\title{
Perturbation Theory for Quantum Computation with Large Number of Qubits
}

\author{
G.P. Berman ${ }^{1}$, G.D. Doolen ${ }^{1}$, D.I. Kamenev ${ }^{1}$, and V.I. Tsifrinovich ${ }^{2}$ \\ 1 Theoretical Division and CNLS, Los Alamos National Laboratory, Los Alamos, NM 87545 \\ 2 IDS Department, Polytechnic University, Six Metrotech Center, Brooklyn, New York 11201
}

We describe a new and consistent perturbation theory for solid-state quantum computation with many qubits. The errors in the implementation of simple quantum logic operations caused by non-resonant transitions are estimated. We verify our perturbation approach using exact numerical solution for relatively small $(L=10)$ number of qubits. A preferred range of parameters is found in which the errors in processing quantum information are small. Our results are needed for experimental testing of scalable solid-state quantum computers.

Pacs numbers: $32.80 . \mathrm{Pj}, 42.50 . \mathrm{Vk}, 05.45 . \mathrm{Mt}$

Several proposals for scalable solid-state quantum computers have been recently published [1] 11]. For the most effective quantum information processing all of these proposals require operations with large number of qubits. In Ref. [12] quantum logic operations between remote qubits were simulated in a chain of $L=1000$ nuclear spins. Such a quantum problem can be solved only using approximate methods since the dimensionality of the Hilbert space increases as $2^{L}$. The approximate procedure used in [12] was based on the selection of states generated as a result of resonant or near-resonant transitions, while the other transitions were neglected in a controlled way. In this Letter we develop a consistent approach to this problem based on perturbation theory. We use our procedure to analytically estimate the probability of generation of unwanted states caused by non-resonant transitions and verify that these agree with the exact numerical solution for the same problem with relatively small number of qubits $(L=10)$, for which the dimensionality of the Hilbert space is not very large $\left(N=2^{10}=1024\right)$. 
Dynamics of a spin chain We consider a chain of identical nuclear spins placed in an external high magnetic field, $B(x)$, which has uniform gradient in the direction of the chain, $x$. The nuclear magnetic resonance (NMR) frequency for the $k$ th spin is $\omega_{k}=\gamma B_{k}$, where $\gamma$ is the nuclear gyromagnetic ratio and $B_{k}$ is the $z$-component of the magnetic field at the location of the $k$ th spin. The gradient of the magnetic field provides a change in $\omega_{k}$ by the value $\delta \omega$ between the neighboring spins. (For physical parameters see Ref. 12].)

The Hamiltonian of the spin chain in an external radio-frequency (rf) field is,

$$
\begin{gathered}
H^{(n)}=-\sum_{k=0}^{L-1} \omega_{k} I_{k}^{z}-2 J \sum_{k=0}^{L-1} I_{k}^{z} I_{k+1}^{z}- \\
\Theta_{n}(t)\left(\Omega_{n} / 2\right) \sum_{k=0}^{L-1}\left[I_{k}^{-} \exp \left(-i\left(\nu_{n} t+\varphi_{n}\right)\right)+I_{k}^{+} \exp \left(i\left(\nu_{n} t+\varphi_{n}\right)\right)\right]=H_{0}+V^{(n)}(t),
\end{gathered}
$$

where $J$ is the Ising interaction constant between neighboring spins and $I_{k}^{z}$ is the operator of the $z$-component of spin $1 / 2 ; \Omega_{n}, \nu_{n}$ and $\varphi_{n}$ are the Rabi frequency, the frequency and the phase of the $n$th pulse, $I_{k}^{ \pm}=I_{k}^{x} \pm I_{k}^{y}$, and $\Theta_{n}(t)$ equals 1 only during the $n$th pulse.

In this Letter we estimate the errors generated during the creation of the entangled state for remote qubits from the ground state of the chain by applying a single $\pi / 2$ pulse and a sequence of $\pi$ pulses in the system described by the Hamiltonian (11). First, we describe schematically the quantum protocol. The first $\pi / 2$ pulse creates a superposition of two states with equal probabilities, $|00 \ldots 00\rangle \rightarrow(1 / \sqrt{2})(|00 \ldots 00\rangle+i|10 \ldots 00\rangle)$. The subsequent pulses, which we describe by the the unitary transformation, $U$, transform this state to an entangled state for remote qubits,

$$
U \frac{1}{\sqrt{2}}(|00 \ldots 00\rangle+i|10 \ldots 00\rangle)=\frac{1}{\sqrt{2}}\left(e^{i \phi_{1}}\left(|00 \ldots 00\rangle+e^{i \phi_{2}}|10 \ldots 01\rangle\right),\right.
$$

where $\phi_{1}$ and $\phi_{2}$ are known phases (see Ref. [12]). The operator $U$ realizes a particular case of the well-known CONTROL-NOT gate. It has the following properties:

$$
\begin{gathered}
U \frac{1}{\sqrt{2}}\left|0_{L-1} 0_{L-2} \ldots 0_{1} 0_{0}\right\rangle=\frac{1}{\sqrt{2}} e^{i \phi_{1}}\left|0_{L-1} 0_{L-2} \ldots 0_{1} 0_{0}\right\rangle, \\
U \frac{1}{\sqrt{2}}\left|1_{L-1} 0_{L-2} \ldots 0_{1} 0_{0}\right\rangle=\frac{1}{\sqrt{2}} e^{i\left(\phi_{2}-\frac{\pi}{2}\right)}\left|1_{L-1} 0_{L-2} \ldots 0_{1} 1_{0}\right\rangle .
\end{gathered}
$$




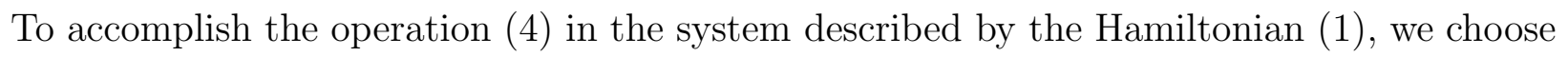
a sequence of $\pi$ pulses with resonant frequencies which will be described elsewhere. If we apply the same protocol to the ground state (operation (33)), then with some probability the system will remain in this state because these pulses have the detunings from resonant transitions, $\Delta_{n} \neq 0$. The near-resonant transitions have the probabilities [12],

$$
\varepsilon_{n}=\left(\Omega_{n} / \lambda_{n}\right)^{2} \sin ^{2}\left(\lambda_{n} \tau_{n} / 2\right)
$$

where $\lambda_{n}=\sqrt{\Delta_{n}^{2}+\Omega_{n}^{2}}$ is the effective field in frequency units and $\tau_{n}$ is the duration of the $n$th pulse. The values of the detunings are the same for all pulses, $\Delta_{n}=\Delta=2 J$, except for the fourth pulse, where $\Delta_{4}=4 J$. In our calculations we assumed $\Omega_{n}=\Omega$ for $n \neq 4$ and $\Omega_{4}=2 \Omega$, so that for all pulses the values of $\varepsilon_{n}$ are the same, $\varepsilon_{n}=\varepsilon$.

We write the wave function, $\Psi(t)$, in the time-interval of the $n$th pulse, in the laboratory system of coordinates in the form,

$$
\Psi(t)=\exp \left[i\left(\nu_{n} t+\varphi_{n}\right) \sum_{k=0}^{L-1} I_{k}^{z}\right] \Psi_{\text {rot }}(t)=\sum_{p} A_{p}(t)|p\rangle \exp \left(-i \chi_{p}^{(n)} t+\xi_{p}^{(n)}\right),
$$

where $\Psi_{\text {rot }}(t)$ is the wave function in a frame rotating with the frequency $\nu_{n} . \chi_{p}^{(n)}=$ $-\left(\nu_{n} / 2\right) \sum_{k=0}^{L-1} \sigma_{k}^{p}, \xi_{p}^{(n)}=\varphi_{n} \sum_{k=0}^{L-1} \sigma_{k}^{p}, \sigma_{k}^{p}=-1$ if the $k$ th spin of the state $|p\rangle$ is in the position 1 and $\sigma_{k}^{p}=1$ if the $k$ th spin is in the position $0,|p\rangle$ is the eigenfunction of the Hamiltonian $H_{0}$. The dynamics during the $n$th pulse with the frequency $\nu_{n}$ is described by the following Schrödinger equation for the coefficients $A_{p}(t)$,

$$
i \dot{A}_{p}(t)=\left(E_{p}-\chi_{p}^{(n)}\right) A_{p}(t)-\frac{\Omega}{2} \sum_{p^{\prime}} A_{p^{\prime}}(t),
$$

where the sum is taken over the states $\left|p^{\prime}\right\rangle$ connected by a single-spin transition with the state $|p\rangle$. Eq. (7]) can be written in the form $i \dot{A}_{p}(t)=\mathcal{H}_{p p^{\prime}}^{(n)} A_{p^{\prime}}(t)$, where $\mathcal{H}_{p p^{\prime}}^{(n)}=H_{p p^{\prime}}^{(n)}-\chi_{p}^{(n)} \delta_{p, p^{\prime}}$, where $\delta_{p, p^{\prime}}$ is the Kronecker $\delta$-symbol.

Under the condition $\Delta \ll \delta \omega$ the energy separation between the $p$ th and the $m$ th diagonal elements of the matrix $H_{p p^{\prime}}^{(n)}$ connected by the resonant or near-resonant transition is $\mid \mathcal{E}_{p}^{(n)}-$ $\mathcal{E}_{m}^{(n)} \mid=\Delta_{n}^{p m}$, where $\mathcal{E}_{p}^{(n)}=E_{p}-\chi_{p}^{(n)}$. This is much less than the energy separation between 
these diagonal elements and diagonal elements of the other states connected with the states $|p\rangle$ and $|m\rangle$ by the non-resonant transitions. In this case one can neglect the interaction of the $p$ th state with all states except the state $|m\rangle$. In this approximation the Hamiltonian matrix $\mathcal{H}_{p p^{\prime}}^{(n)}$ breaks up into $N / 2$ approximately independent matrices $2 \times 2$, where $N=2^{L}$.

Errors in the creation of an entangled state for remote qubits. The matrix approach allows us to estimate errors in the logic gate (2) caused by near-resonant and non-resonant transitions. Suppose that initially the eigenstate $|p\rangle$ of the Hamiltonian $H_{0}$ is populated. We want to calculate the probability of non-transition to the state $|r\rangle$ with $\left|E_{p}-E_{r}\right| \sim \delta \omega$, where the states $E_{p}$ and $E_{r}$ are connected by a flip of $k^{\prime}$ th spin, whose NMR frequency differs by the value $\sim \delta \omega$ from the frequency of the resonant transition. Since the matrix elements are small, we can write

$$
\psi_{q}^{(n)}=\psi_{q}^{0(n)}+\sum_{p}^{\prime} \frac{v_{q p}^{(n)}}{\mathcal{E}_{q}^{(n)}-\mathcal{E}_{p}^{(n)}} \psi_{p}^{0(n)},
$$

where prime in the sum means that the term with $p=q$ is omitted; $v_{q p}^{(n)}$ is the matrix element for transition between the states $\psi_{q}^{0(n)}$ and $\psi_{p}^{0(n)}$.

Because the matrix $\mathcal{H}_{q p}^{(n)}$ is divided into $2^{L-1}$ relatively independent $2 \times 2$ blocks, the wave function, $\psi_{p}^{0(n)}$, in Eq. (8) is an eigenfunction of a single block $2 \times 2$ with all other elements being equal to zero. In the case for which the two states $\left|p^{\prime}\right\rangle$ and $|p\rangle$ are connected by a near-resonant transition, the function, $\psi_{p}^{0(n)}$, in Eq. (8) has the form, $\psi_{p}^{0(n)} \approx[1-$ $\left.\left(\Omega^{2} / 8 \Delta^{2}\right)\right]|p\rangle+(\Omega / 2 \Delta)\left|p^{\prime}\right\rangle$. On the other hand, if the states $|p\rangle$ and $\left|p^{\prime}\right\rangle$ are connected by an exact resonant transition, we have $\psi_{p}^{0(n)}=(1 / \sqrt{2})\left(|p\rangle+\left|p^{\prime}\right\rangle\right)$ and $\psi_{p^{\prime}}^{0(n)}=(1 / \sqrt{2})\left(|p\rangle-\left|p^{\prime}\right\rangle\right)$. In both cases the probability of non-resonant transition from the state $|q\rangle$ to the state $|p\rangle$ connected with the state $|q\rangle$ by flip of the $k^{\prime}$ th spin (up to the value $\mu=(\Omega / \omega)^{2}$ ) is

$$
P_{p q}^{(n)}=\left|\left\langle p \mid \psi_{q}\right\rangle\right|^{2}=\left(\frac{V_{p q}^{(n)}}{\mathcal{E}_{q}^{(n)}-\mathcal{E}_{p}^{(n)}}\right)^{2} \approx\left(\frac{\Omega_{n}}{2\left|k_{n}-k^{\prime}\right| \delta \omega}\right)^{2}
$$

where $\left|k_{n}-k^{\prime}\right|$ is the distance from the $k^{\prime}$ th spin (whose NMR frequency, $\omega_{k^{\prime}}$, is non-resonant) to the $k_{n}$ th spin with resonant (or near-resonant) NMR frequency.

The probability $\mu_{L-1}$ (here the subscript of $\mu$ stands for the number of the resonant 
spin) of generation of unwanted states by the first $\pi / 2$ pulse in the result of non-resonant transitions is

$$
\mu_{L-1}=\left(\frac{\Omega}{2 \delta \omega}\right)^{2} \sum_{k^{\prime}=0}^{L-2} \frac{1}{\left|L-1-k^{\prime}\right|^{2}} .
$$

After the first $\pi / 2$ pulse, the probability of the correct procedure in implementation of the logic gate is $P_{1}=1-\mu_{L-1}$. The probability of correct implementation of the operation (2) by applying $2 L-2$ pulses is

$$
\begin{gathered}
P_{2 L-2}=\frac{1}{2}\left(1-\mu_{L-1}\right)\left(1-\mu_{L-2}-\varepsilon\right)\left(1-4 \mu_{L-2}-\varepsilon\right)\left(1-\mu_{0}-\varepsilon\right) \prod_{i=1}^{L-3}\left(1-\mu_{i}-\varepsilon\right)^{2}+ \\
\frac{1}{2}\left(1-\mu_{L-2}\right)\left(1-4 \mu_{L-2}\right) \prod_{i=0}^{L-3}\left(1-\mu_{i}\right)^{2},
\end{gathered}
$$

where the first term is provided by the operation (3), and the last term is due to (4).
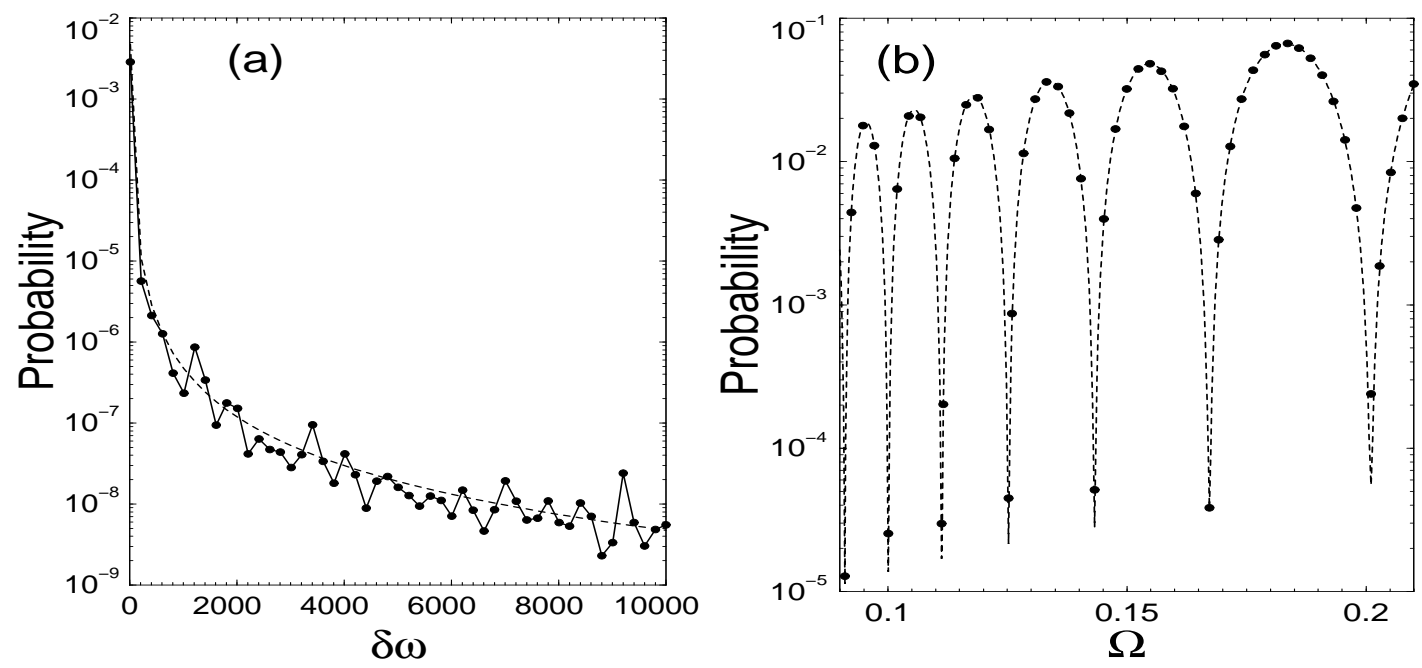

FIG. 1. (a) The probability, $\mathcal{P}$, of generation of unwanted states in implementation of the logic gate (2) at $\varepsilon=0$. Filled circles connected by the solid line are the numerical results, dashed line is the analytic estimate; $\Omega=2 J / \sqrt{\left(4 k^{2}-1\right)}, k=8$. (b) $\mathcal{P}$ as a function of $\Omega$. Filled circles are the numerical results. The dashed line is the analytic estimate, $\delta \omega=100 ; J=1, L=10, \varphi_{n}=0$ for all $n$.

Numerical results. In Fig. 1 (a) we compare the total probability, $\mathcal{P} \equiv 1-P_{2 L-2}$, of generation of unwanted states with the result of exact numerical solution of Eq. (7) for 
the case in which the probability of near-resonant transitions is negligibly small, i.e. when $\varepsilon=0$. From Fig. 1 (a) one can see that in this situation $\mathcal{P}$ decreases as $\delta \omega$ increases.

When $\varepsilon$ is large, $\varepsilon \gg \mu$, and $\Delta \ll \delta \omega$, the probability of generation of unwanted states is mostly defined by the value of $\varepsilon$ and is almost independent of $\delta \omega$. In Fig. 1 (b) we plot the probability $\mathcal{P}$ as a function of $\Omega$. The value of $\delta \omega$ fixes the values of the minima, $\mathcal{P}_{\min }$, in Fig. 1 (b): for larger $\delta \omega$ the minima in the plot in Fig. 1 (b) become deeper. The values of different minima in Fig. 1 (b) indicate the contribution of non-resonant processes to the probability $\mathcal{P}$. Since the value of $\Omega$ in Fig. 1 (b) does not change significantly, the contribution of non-resonant processes to the probability of errors is approximately the same for all $\Omega$ and is equal to $\mathcal{P}_{\min }$. One can see that this contribution is negligibly small in comparison with the contribution of near-resonant processes (defined by $\varepsilon$ ) for all $\Omega$, except for the small regions of $\Omega$, where $\varepsilon$, defined by Eq. (5), is minimal.
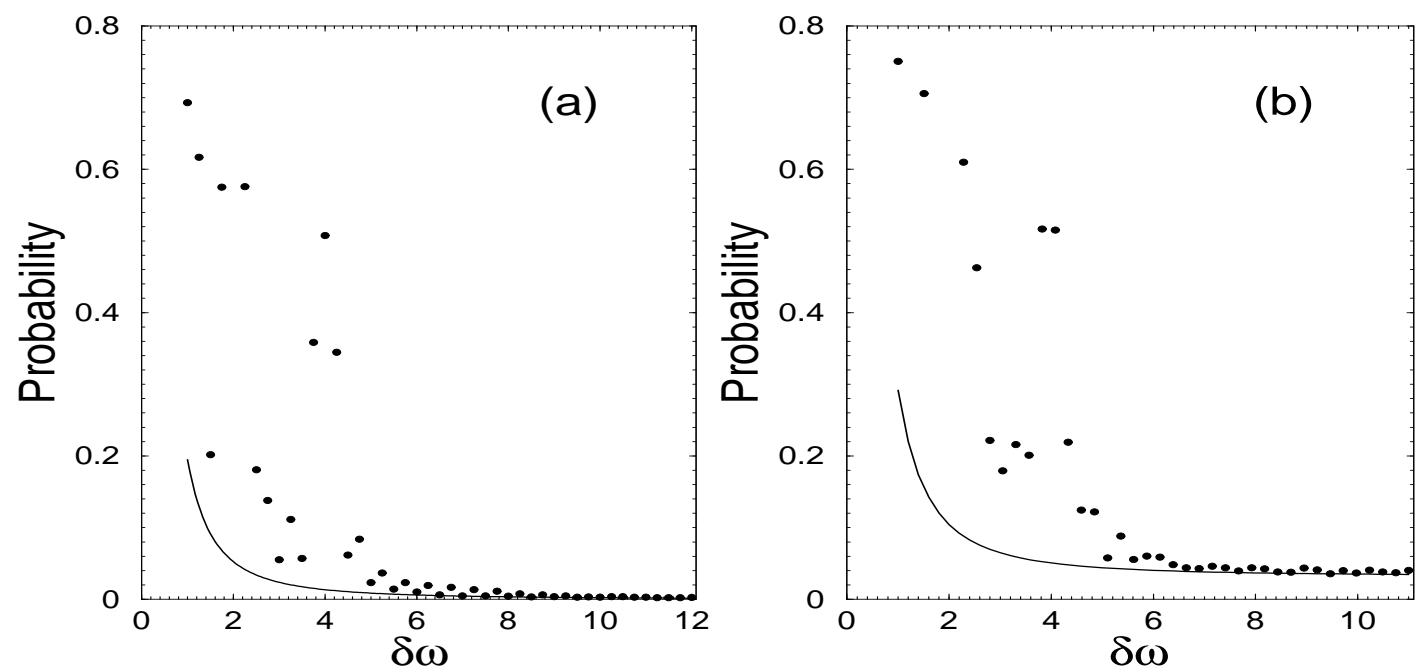

FIG. 2. The total probability of unwanted states in implementation of the logic gate (2) as a function of $\delta \omega$ when the value of $\delta \omega$ is comparable with the value of the detuning, $\Delta$. (a) $\Omega=2 J / \sqrt{4 k^{2}-1}, \mathrm{k}=8(\varepsilon=0),(\mathrm{b}) \Omega=0.15(\varepsilon=0.0039)$. Filled circles are the numerical results, solid line is the analytical estimate for $\mathcal{P} ; J=1, L=10, \varphi_{n}=0$ for all $n$.

When the perturbation parameters of the problem, $\varepsilon$ and $\mu$, are small, using Eq. (11) one can easily estimate the probability of generation of unwanted states in the implementation of the logic gate (2) when the number of qubits in the spin chain is large (for example, for 
$L \sim 1000$ ). In this case the Eq. (11) is important for estimation of errors, since the exact solution of the problem requires diagonalization of enormous matrices of size $2^{L} \times 2^{L}$.

We should note that one more condition (except for $\varepsilon, \mu \ll 1$ ) must be satisfied for Eq. (11) to be valid. The value of detuning, $\Delta$, should be small in comparison with the difference between NMR frequencies of the spins, $\Delta \ll \delta \omega$. In Figs. 2 (a) and (b) we plot the probability, $\mathcal{P}$, as a function of $\delta \omega$ for small and large values of $\varepsilon$. One can see that our results are valid only when $\Delta \ll \delta \omega$, in spite of the fact that the parameter $\Delta / \delta \omega$ does not appear explicitly in Eq. (11). From Fig. 2 (b) one can see that the probability of unwanted states, $\mathcal{P}$ for $\varepsilon \gg \mu$ and for large $\delta \omega$ becomes relatively independent of $\delta \omega$. In this case the value of $\mathcal{P}$ is defined by $\varepsilon$ which, due to Eq. (5), does not depend on $\delta \omega$.

This work was supported by the Department of Energy (DOE) under contract W-7405ENG-36, by the National Security Agency (NSA), and by the Advanced Research and Development Activity (ARDA).

[1] A. Barenco, D. Deutsch, A. Ekert, and R. Jozsa, Phys. Rev. Lett. 74, 4083 (1995).

[2] B.E. Kane, Nature 393, 133 (1998).

[3] Y. Nakamura, Yu. A. Pashkin, and J. S. Tsai, Nature 398, 786 (1999).

[4] Y. Makhlin, G. Schön, and A. Shnirman, Nature 398, 305 (1999).

[5] F. Yamaguchi and Y. Yamamoto, Microelectron. Eng. 47, 273 (1999).

[6] M. Sherwin, A. Imamoglu, and T. Montroy, quant-ph/9905096.

[7] A. Imamoglu, D.D. Awschalom, G. Burkard, D.P. DiVincenzo, D. Loss, M. Sherwin, and A. Small, Phys. Rev. Lett. 83, 4204 (1999).

[8] R. Vrijen, E. Yablonovitch, K. Wang, H.W. Jiang, A. Balandin, V. Roychowdhury, T. Mor, and D. DiVincenzo, Phys. Rev. A 62, 2306 (2000). 
[9] G.P. Berman, G.D. Doolen, P.C. Hammel, and V.I. Tsifrinovich, Phys. Rev. B 61, 14694 (2000).

[10] G.P. Berman, G.D. Doolen, P.C. Hammel, and V.I. Tsifrinovich, Phys. Rev. Lett. 86, 2894 (2001).

[11] G.P. Berman, G.W. Brown, M.E. Hawley, and V.I. Tsifrinovich, quant-ph/0103008.

[12] G.P. Berman, G. D. Doolen, G. V. Lòpez, and V. I. Tsifrinovich, Phys. Rev. A 61, 2307 (2000). 